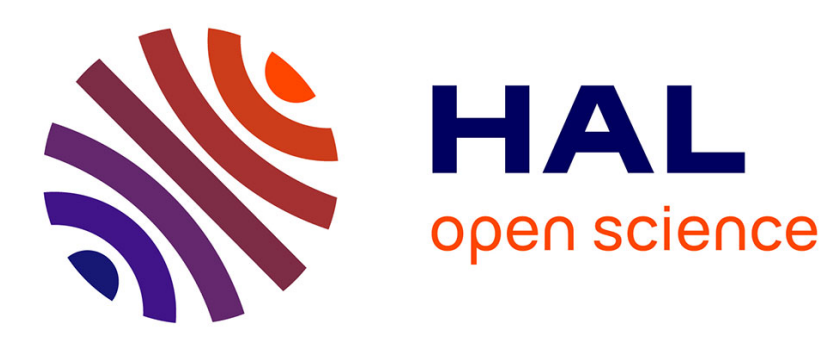

\title{
Uncertainty analysis of river flooding and dam failure risks using local sensitivity computations
}

Carole Delenne, B Cappelaere, V Guinot

\section{To cite this version:}

Carole Delenne, B Cappelaere, V Guinot. Uncertainty analysis of river flooding and dam failure risks using local sensitivity computations. Reliability Engineering and System Safety, 2012, 107, pp.171-183. 10.1016/j.ress.2012.04.007 . hal-01196887

\section{HAL Id: hal-01196887 \\ https://hal.science/hal-01196887}

Submitted on 10 Sep 2015

HAL is a multi-disciplinary open access archive for the deposit and dissemination of scientific research documents, whether they are published or not. The documents may come from teaching and research institutions in France or abroad, or from public or private research centers.
L'archive ouverte pluridisciplinaire HAL, est destinée au dépôt et à la diffusion de documents scientifiques de niveau recherche, publiés ou non, émanant des établissements d'enseignement et de recherche français ou étrangers, des laboratoires publics ou privés. 


\title{
Uncertainty analysis of river flooding and dam failure risks using local sensitivity computations.
}

\author{
C. Delenne $e^{\mathrm{a}, *}$, B. Cappelaere ${ }^{\mathrm{b}}$, V. Guinot ${ }^{\mathrm{a}}$ \\ ${ }^{a}$ Université Montpellier 2, UMR HydroSciences Montpellier, 34095 Montpellier, France. \\ ${ }^{b}$ Insitut de Recherche et Développement, UMR HydroSciences Montpellier, 34095 Montpellier, France.
}

\begin{abstract}
The potential of Local Sensitivity Analysis (LSA) for analysis of uncertainty with respect to two major risks in river hydrodynamics - flash flood and dam failure - is assessed. LSA, implemented as an equation-based method, is compared to a Global Uncertainty Analysis (GUA) consisting in running Monte Carlo simulations with a hydrodynamic model. For a given statistical distribution of the model input parameters, the mean and standard deviation of the output variables are estimated with the two methods. In all single or multiple parameter cases investigated, including as much as $\pm 80 \%$ relative variation, LSA provides similar results to GUA, while requiring only one simulation instead of several hundreds or thousands. Only within a few meters of the shock (flow discontinuity) generated by the breaking of a dam do the two methods depart. This paper shows that despite the nonlinearity of river flow processes, the first order, local approach remains generally valid for uncertainty analysis of hydrodynamic risks, even in the case of large parameter uncertainty. The contrast in importance of the various parameters on both sides of a shock is also highlighted.
\end{abstract}

Keywords: uncertainty analysis, shallow water equations, river flood, dam-break, global approach, local approach.

\section{Introduction}

Modelling real-world phenomena, especially when they are extreme, is generally plagued with errors arising from many approximations. A mathematical model schematizes a physical process, using equations that are solved approximately by numerical methods; it requires several input factors to be measured or estimated, and parameters to be calibrated, all operations that bring substantial uncertainties into the model. Uncertainty analysis is thus needed to assess the reliability of model predictions, accounting for multiple sources of uncertainty in model design and implementation. Providing prediction uncertainty concurrently with model outputs should be considered as an integral part of the modelling job [26], but it is often disregarded for lack of readily applicable, computer and human resources-parsimonious procedures.

In the field of water engineering and management, uncertainty and sensitivity analyses are of high importance, when dealing for instance with resource allocation, water quality [10], hazards such as flood, tidal wave or dam/dike break(e.g. $[8,15,24,23])$, or impacts of climate change on hydrological systems $[18,22,28]$. For instance, uncertainty arising from model calibration is increasingly handled using the empirical, sampling-based Generalised

\footnotetext{
* Corresponding author

Email addresses: delenne@msem.univ-montp2.fr (C. Delenne), bernard.cappelaere@mpl.ird.fr (B. Cappelaere), guinot@msem.univ-montp2.fr (V. Guinot)
} 
Likelihood Uncertainty Evaluation (GLUE) methodology [2, 3], developed in the hydrological modelling context.

Sampling-based techniques, that resort to extensive exploration of the space of possible model inputs with ensuing multiple model runs, are a common approach. An advantage is that no specific analysis or prior assumption needs to be made on the model itself, seen as a black box. When inputs uncertainties can be described statistically by means of probability distribution functions, Monte Carlo simulation yields a statistical description of uncertain model outputs. However, the combination of commonly encountered input space dimensions and model run times often makes this approach hardly practicable. Numerical models often require a large number of input parameters, and inputs may not be scalars but space or time functions, leading to excessive sample sizes due to the so-called curse of dimensionality. Substantial research has been devoted over the last decades to reducing computing requirements of Monte Carlo techniques, e.g. through better sampling procedures (e.g. [21, 16]) or meta-model development (e.g. [17, 9]). However computational limits remain an obstacle to generalizing the method for computationally expensive simulations.

When the model can be considered as nearly linear in the subspace of possible input values, first-order estimates of uncertainty propagation through the model can be readily obtained from computation of local sensitivities, i.e., partial derivatives of the model for a single set of inputs. Estimating local sensitivity is a relatively widespread requirement in model development and operation, for it is very helpful for model calibration or validation for instance [11, 20], and can be achieved with limited additionnal computational time. The empirical, finite-difference approach is frequently used, consisting in computing the difference in output between two simulations with two slightly different values of the parameter of interest, normalised by the parameter variation. More powerful and promising is the equation-based method that consists in deriving the so-called tangent linear model. This augments the original model with the governing equations for propagation of sensitivities along the model variables' trajectory. Using the local sensitivity approach obviously generates difficulties when the model output becomes discontinuous [1, 14, 13]. However, when they exist, discontinuities are in many cases limited to very specific locations in the model space, and many seemingly nonlinear models actually display quasi-linear behaviour over most of their working space. Other approaches to sensitivity analysis exist for expressing the relative dependencies of model outputs on uncertain inputs. In particular, quantitative global sensitivity indices have been devised to inform about the relative shares of the various inputs in producing the output uncertainty [4]. These include variance-oriented indices $[27,25]$ or moment-independent indices [5]. Unfortunately these very informative, global procedures suffer, to an even greater extent, from the aforementioned difficulties with sampling-based techniques, let aside the additional algorithmic complexity, which makes them topics of on-going research and currently limits their diffusion among modellers.

The purpose of this paper is to investigate the suitability of the local sensitivity approach to uncertainty analysis (referred to as LSA in the following) in the modelling of major, transient-state hydrodynamic risks such as propagation of flash floods in a watercourse or failure of a dam. These two major issues for watershed development and risk prevention can be handled with the one-dimensional Shallow Water model for open channel flow, a system of partial differential equations that is generally viewed as being largely non linear. This model requires parameters and forcing inputs that can be either impossible to measure, hence requiring calibration, or estimated only with high uncertainty. It is thus important to assess the propagation of these input uncertainties to the model's output variables, namely the time-space variations of water depth and velocity (or discharge) of water flow. A direct, equation-based tangent linear model was developed for the 1-D Shallow Water model to compute model sensitivities by $[12,7]$.

The structure of this paper is as follows. Section 2 defines the general methodology used for uncertainty analysis, using successively a Monte Carlo based Global Uncertainty Analysis 
(GUA), and a Local Sensitivity Analysis (LSA). Section 3 gives the theoretical background for the flow model and the local equation-based sensitivity approach. Governing equations for the flow and the sensitivity are introduced as well as the numerical method used to solve them. Section 4 presents the two application problems, together with corresponding expressions of the source terms and boundary conditions for the sensitivity model depending on the parameter of interest. Section 5 is devoted to the application of the LSA and GUA methods to these problems, and to comparing their results in a one-factor-at-a-time (OAT) study as well as for the simultaneous variation of the main three parameters in the case of the dam-break problem.

\section{Uncertainty analysis methodology}

To apply the one-dimensional shallow water equations of open channel flow, (section 3) to the study of flash flood and dam failure risks, the space distribution of geometric (bottom slope) and hydrodynamic (friction coefficient) parameters along a river reach of length $L$, as well as the initial and boundary conditions (in particular: initial water depths, discharge variations at the upstream boundary) are required. The model output is $\mathbf{U}(x, t)=[h, q]^{T}$, where the water depth $h$ and the unit discharge $q$ are functions of space and time (see section 3).

Given a statistical distribution of one or several uncertain model inputs, model output uncertainty is estimated in this study as the first two statistical moments of the variables, i.e. their means and standard deviations. These moments are estimated both with the MonteCarlo technique (GUA), based on parameter sampling and multiple model runs, and with the first-order, local sensitivity-based approximation (LSA) of the first output moments (subscripts ${ }_{G}$ and $L$ are used to denote moments estimated with the GUA and the LSA methods, respectively). The model output considered here for the analysis is the spacedependent solution $\mathbf{U}(x)$ after a given simulation time $T_{\max }$. Symbols and notations are defined in Appendix A.

\subsection{Parameters distribution and global (GUA) estimation of model uncertainty}

Parameter uncertainty is modelled using a Beta distribution, which has the following probability density function

$$
f(\phi ; \alpha, \beta)=\frac{\phi^{\alpha-1}(1-\phi)^{\beta-1}}{\int_{0}^{1} u^{\alpha-1}(1-u)^{\beta-1} \mathrm{~d} u}
$$

where $\phi \in[0,1]$ and $(\alpha, \beta)$ are shape parameters taken here as $\alpha=\beta=5$ (symmetric distribution). Unlike the widely used Gaussian distribution, this function has a finite support, thereby avoiding possible non-physical values of the parameters (such as negative values for the friction coefficient). The distribution obtained for $\phi$ is then centered onto the nominal value $\psi^{0}$ of the parameter of interest $\psi$ and rescaled to a min-max interval: $\left[\psi^{0}-\Delta \psi ; \psi^{0}+\Delta \psi\right]$. Monte Carlo simulations are performed using a sample of the parameter $\psi$ generated by a random sample of size $N$ drawn from a Beta distribution.

When several parameters are considered uncertain simultaneously, they are taken independent. Model uncertainty is assessed for the global, GUA approach as the sample mean $\left(\mu_{\mathbf{U}}(x)\right)_{G}$ and standard deviation $\left(\sigma_{\mathbf{U}}(x)\right)_{G}$ of the $N$ model outputs $\mathbf{U}(x)_{n}, n=1 \ldots N$.

\subsection{First-order, local (LSA) estimation of model uncertainty}

The parameters distribution being assumed symmetrical, the model output produced with the mean, i.e. nominal parameter set provides an estimation with first-order accuracy of the output mean, denoted $\left(\mu_{\mathbf{U}}(x)\right)_{L}$. In the case when one parameter $\psi$ is allowed to vary at a time, the standard deviation $\sigma_{\mathbf{U}}(x)$ of the model output at each abscissa $x$ can 
be first-order estimated from the sensitivity solution $\mathbf{s}=(\eta, \theta)^{\mathrm{T}}$ obtained with the nominal parameter set, as $\left(\sigma_{\mathbf{U}}(x)\right)_{L}=|\mathbf{s}(x)| \sigma_{\psi}$, i.e.:

$$
\begin{aligned}
\left(\sigma_{h}(x)\right)_{L} & =\left|\eta_{\psi}(x)\right| \sigma_{\psi} \\
\left(\sigma_{q}(x)\right)_{L} & =\left|\theta_{\psi}(x)\right| \sigma_{\psi}
\end{aligned}
$$

where $\sigma_{\psi}$ is the standard deviation of the input distribution of the parameter $\psi$.

In the case when $N$ parameters vary at the same time and independently, the first-order estimate of standard deviation writes:

$$
\begin{aligned}
\sigma_{h L} & =\left(\sum_{i=1}^{N} \eta_{\psi_{i}}^{2} \sigma_{\psi_{i}}^{2}\right)^{1 / 2} \\
\sigma_{q L} & =\left(\sum_{i=1}^{N} \theta_{\psi_{i}}^{2} \sigma_{\psi_{i}}^{2}\right)^{1 / 2}
\end{aligned}
$$

where $\eta_{\psi_{i}}$ (resp. $\theta_{\psi_{i}}$ ) is the sensitivity of $h$ (resp. $q$ ) with respect to parameter $\psi_{i}$, and $\sigma_{\psi_{i}}^{2}$ is the variance of the input distribution for the parameter $\psi_{i}$.

\subsection{Comparison of GUA and LSA estimates of moments}

The two methods are compared through their estimations of the model output moments, $\mu$ and $\sigma$. Taking the global (GUA) estimates $\mu_{G}$ and $\sigma_{G}$ as the reference, average relative errors are computed for each moment as:

$$
\begin{aligned}
\varepsilon_{\mu} & =\frac{\Delta x}{L} \sum \frac{\left|\mu_{G}-\mu_{L}\right|}{\mu_{G}} \\
\varepsilon_{\sigma} & =\frac{\Delta x}{L} \sum \frac{\left|\sigma_{G}-\sigma_{L}\right|}{\sigma_{G}}
\end{aligned}
$$

where $\Delta x$ is a spatial discretization step along the length $\mathrm{L}$ of river reach, and the summation applies to the resulting space steps.

The flow and first-order sensitivity models are briefly recalled in the following section and applied to the flood propagation and dam-break problems in section 4. Comparison of the LSA and GUA is performed in section 5 .

\section{Flow and sensitivity models}

\subsection{Governing equations}

The one-dimensional Shallow Water model of open channel flow can be written in vector form as the following system of hyperbolic, partial differential equations [6]:

$$
\frac{\partial \mathbf{U}(x, t)}{\partial t}+\frac{\partial \mathbf{F}(x, t)}{\partial x}=\mathbf{S}(x, t)
$$

where $t$ is time and $x$ the abscissa along the flow direction, and where the conserved variable $\mathbf{U}$, the flux vector $\mathbf{F}$ and the source term $\mathbf{S}$ are defined as

$$
\mathbf{U}=\left[\begin{array}{c}
h \\
q
\end{array}\right], \quad \mathbf{F}=\left[\begin{array}{c}
q \\
\frac{q^{2}}{h}+g \frac{h^{2}}{2}
\end{array}\right], \quad \mathbf{S}=\left[\begin{array}{c}
0 \\
g h\left(S_{0}-S_{f}\right)
\end{array}\right]
$$

with $h$ the water depth, $q$ the unit discharge (i.e., the discharge per unit width), $g$ the gravitational acceleration, $S_{0}$ the bottom slope and $S_{f}$ the friction slope. For the sake of 
conciseness, the friction slope is assumed to obey a classical Manning-Strickler law [6] under the wide channel approximation:

$$
S_{f}=q|q| n_{M}^{2} h^{-10 / 3}
$$

where $n_{M}$ is Manning's friction coefficient. Solving Eq. (6) requires the knowledge of the space-dependent initial condition $\mathbf{U}(x, t=0)$, as well as two scalar, time-dependent boundary conditions $f_{b}\left(U\left(x_{b}, t\right), t\right)=0$, where subscript ${ }_{b}$ refers to the upstream or the downstream boundary.

The governing equations for sensitivity are obtained by differentiating the flow equations (6) with respect to a given parameter $\psi(x, t)$, where $\psi$ represents any one of the $S_{0}$ or $S_{f}$ parameters or of the prescribed inputs in the initial or boundary conditions (see for example [? 12] for a detailed description of the method):

$$
\begin{gathered}
\frac{\partial \mathbf{s}(x, t)}{\partial t}+\frac{\partial \mathbf{G}(x, t)}{\partial x}=\mathbf{Q}(x, t) \\
\mathbf{s}=\left[\begin{array}{c}
\eta \\
\theta
\end{array}\right] \equiv\left[\begin{array}{c}
\partial h / \partial \psi \\
\partial q / \partial \psi
\end{array}\right] \\
\mathbf{G}=\frac{\partial \mathbf{F}}{\partial \mathbf{U}} \mathbf{s}=\left[\begin{array}{cc}
0 & 1 \\
c^{2}-u^{2} & 2 u
\end{array}\right] \mathbf{s}=\left[\begin{array}{c}
\theta \\
\left(c^{2}-u^{2}\right) \eta+2 u \theta
\end{array}\right] \\
\mathbf{Q}=\frac{\partial \mathbf{S}}{\partial \mathbf{U}} \mathbf{s}+\frac{\partial \mathbf{S}}{\partial \psi} \varepsilon-\frac{\partial}{\partial x}\left(\frac{\partial \mathbf{F}}{\partial \psi} \varepsilon\right)
\end{gathered}
$$

where $c=(g h)^{1 / 2}$ is the propagation speed of the waves in the fluid at rest, $u$ is the flow velocity and $\varepsilon(x, t)$ is the so-called perturbation indicator, with $\varepsilon=0$ in the regions where the parameter remains unchanged and $\varepsilon=1$ in the regions where $\psi$ is perturbed for the sensitivity analysis. Eqs. (9) are the conservation form of the Shallow Water Sensitivity Equations. The source term $\mathbf{Q}$ defined in Eq. (9d) is the only one in Eq (9) that depends on the nature of the parameter $\psi$ and will be explicited in section 4 for each application presented. Also varying with parameter $\psi$ and developed in section 4 are the expressions of the initial and boundary conditions, which are paired with those of the flow model.

\subsection{Numerical method}

In the general case, for which no analytical solution can be found, the flow and sensitivity equations are discretized using a finite volume formulation [12]:

$$
\begin{aligned}
& \mathbf{U}_{i}^{n+1}=\mathbf{U}_{i}^{n}-\frac{\Delta t}{\Delta x_{i}}\left(\mathbf{F}_{i-1 / 2}^{n+1 / 2}-\mathbf{F}_{i+1 / 2}^{n+1 / 2}\right)+\Delta t \mathbf{S}_{i}^{n+1 / 2} \\
& \mathbf{s}_{i}^{n+1}=\mathbf{s}_{i}^{n}-\frac{\Delta t}{\Delta x_{i}}\left(\mathbf{G}_{i-1 / 2}^{n+1 / 2}-\mathbf{G}_{i+1 / 2}^{n+1 / 2}\right)+\Delta t \mathbf{Q}_{i}^{n+1 / 2}
\end{aligned}
$$

where $\Delta t$ is the computational time step, $\Delta x_{i}$ is the width of the cell $i, \mathbf{U}_{i}^{n}$ and $\mathbf{s}_{i}^{n}$ are respectively the average value of $\mathbf{U}$ and $\mathbf{s}$ over the cell $i$ at the time level $n, \mathbf{F}_{i-1 / 2}^{n+1 / 2}$ and $\mathbf{G}_{i-1 / 2}^{n+1 / 2}$ are the average values of the fluxes $\mathbf{F}$ and $\mathbf{G}$ through the interface $i-1 / 2$ (between the cells $i-1$ and $i$ ) between the time levels $n$ and $n+1, \mathbf{S}_{i}^{n+1 / 2}$ and $\mathbf{Q}_{i}^{n+1 / 2}$ are the average values of $\mathbf{S}$ and $\mathbf{Q}$ over the cell $i$ between the time levels $n$ and $n+1$.

The fluxes $\mathbf{F}$ and $\mathbf{G}$ in Eqs (10) are computed by solving the following Riemann problems using the HLLC solver (see e.g. [? ] for more details):

$$
\frac{\partial \mathbf{U}}{\partial t}+\frac{\partial \mathbf{F}}{\partial x}=0
$$




$$
\begin{gathered}
\frac{\partial \mathbf{s}}{\partial t}+\frac{\partial \mathbf{G}}{\partial x}=0 \\
(\mathbf{U}, \mathbf{s})(x, 0)= \begin{cases}\left(\mathbf{U}_{\mathrm{L}}, \mathbf{s}_{\mathrm{L}}\right) & \text { for } x<x_{i+1 / 2} \\
\left(\mathbf{U}_{\mathrm{R}}, \mathbf{s}_{\mathrm{R}}\right) & \text { for } x \geq x_{i+1 / 2}\end{cases}
\end{gathered}
$$

where $x_{i+1 / 2}$ is the abscissa of the interface.

\section{Investigated problems}

Two different types of fluvial risk situations are analysed. The first is the frequently encountered case of propagation of a transient flood discharge signal from the upstream catchment along a channel reach. The second is the more challenging, extreme case of the sudden collapsing of a dam. Simplified configurations are considered to investigate these two problems.

\subsection{Flood propagation}

To investigate this problem, a triangular flood hydrograph is prescribed at the upstream boundary of a uniform channel reach (see Figure 1). The discharge is first taken constant at $q_{\min }$, during a time $t_{1}$ sufficient for the simulation from an initial condition at rest to reach the steady state. Then, the flood rises to its maximum $q_{\max }$ at $t_{2}$ and decreases to recover its initial value $q_{\min }$ at $t_{3}$. The prescribed unit discharge $q_{\text {up }}$ at the upstream boundary thus writes:

$$
q_{\mathrm{up}}(t)= \begin{cases}q_{\min } & t<t_{1} \\ q_{\min }+\frac{q_{\max }-q_{\min }}{t_{2}-t_{1}}\left(t-t_{1}\right) & t \in\left[t_{1} ; t_{2}\right] \\ q_{\max }+\frac{q_{\min }-q_{\max }}{t_{3}-t_{2}}\left(t-t_{2}\right) & t \in\left[t_{2} ; t_{3}\right] \\ q_{\min } & t>t_{3}\end{cases}
$$

It is assumed that a sill at the downstream boundary imposes a nearly critical regime, leading to the following height-discharge relationship:

$$
\left(\frac{u}{\sqrt{g h}}\right)_{\mathrm{ds}}=\mathrm{F}_{\mathrm{ds}}
$$

where $F_{\mathrm{ds}}$ is the Froude number chosen close to but smaller than unity so that the flow regime remains subcritical.

The sensitivity analysis is performed with respect to the maximum input discharge $q_{\max }$. Hence, the prescribed input sensitivity $\theta$ at the upstream boundary is also a triangular function, with a maximum of one when the peak discharge enters the domain (see Figure1):

$$
\theta_{\text {up }}(t)= \begin{cases}0 & t<t_{1} \\ \frac{t-t_{1}}{t_{2}-t_{1}} & t \in\left[t_{1} ; t_{2}\right] \\ \frac{t_{3}-t}{t_{3}-t_{2}} & t \in\left[t_{2} ; t_{3}\right] \\ 0 & t>t_{3}\end{cases}
$$

At the downstream boundary, the sensitivity relationship is obtained by differentiating equation (13) with respect to the investigated parameter:

$$
\left[\frac{1}{\sqrt{g h}}\left(\frac{\theta}{h}-\frac{3 q}{2 h^{2}} \eta\right)\right]_{\mathrm{ds}}=0
$$




\subsection{Dam-break problem}

The dam-break problem [29] has been widely used as a validation test case for Shallow Water Equations solvers. It is a simplified representation of the breaking of a dam as the sudden release of the reservoir water consecutive to instantaneous, total dam collapse. In the initial system considered here (Figure 2), the water is at rest inside the reservoir whereas a uniform flow is assumed in the downstream river, without loss of generality.

The dam-break problem is an initial value problem the initial conditions of which can be written as:

$$
\begin{gathered}
z(x, 0)= \begin{cases}z_{\mathrm{L}}=h_{\mathrm{L}}+z_{b} & \text { for } x<x_{0} \\
z_{\mathrm{R}}=h_{\mathrm{R}}+z_{b} & \text { for } x>x_{0}\end{cases} \\
q(x, 0)= \begin{cases}0 & \text { for } x<x_{0} \\
u_{\mathrm{R}} h_{\mathrm{R}} & \text { for } x>x_{0}\end{cases}
\end{gathered}
$$

where $z$ is the water elevation, $z_{b}$ is the bottom elevation, $x_{0}$ is the abscissa of the dam and $u_{\mathrm{R}}$ is the initial flow velocity prescribed downstream in the river. In the following, the initial water depth $h_{R}$ in the river will be taken either as any prescribed arbitrary value, or as the so-called normal depth $h_{N}$ defined by considering the friction slope $S_{f}$ equal to the bottom slope $S_{0}$ in Eq. (8), leading to:

$$
h_{N}=\left(\frac{u_{\mathrm{R}} n_{M}}{\sqrt{S_{0}}}\right)^{3 / 2}
$$

As shown in Appendix B, an analytical solution for the flow (6) and sensitivity (9) equations can be obtained for the dam-break problem when no friction and bottom slope are considered.

The sensitivity analysis of the dam-break problem will be performed with respect to the main three parameters: the initial water elevation $z_{\mathrm{L}}$ in the reservoir, the Manning's friction coefficient $n$ and the bottom slope $S_{0}$. The expression of the sensitivity source term $\mathbf{Q}$ (Eq. 9d) and initial and boundary conditions are given in the following paragraphs depending on the parameter of interest.

1. Considering the sensitivity to an initial condition, such as the initial water elevation in the reservoir $h_{\mathrm{L}}$, yields

$$
\varepsilon(x, t)=0 \quad \forall t>0
$$

The expression of $\mathbf{Q}$ then reduces to the term $(\partial \mathbf{S} / \partial \mathbf{U}) \mathbf{s}$ which writes

$$
\frac{\partial \mathbf{S}}{\partial \mathbf{U}} \mathbf{S}=\left(\begin{array}{c}
0 \\
g\left(S_{0}+7 / 3 S_{f}\right) \eta-2 g n_{M}^{2}|q| h^{-7 / 3} \theta
\end{array}\right)
$$

The initial conditions in sensitivity for $\psi=h_{\mathrm{L}}$ are given by

$$
\begin{gathered}
\eta(x, 0)= \begin{cases}1 & \text { for } x<x_{0} \\
0 & \text { for } x>x_{0}\end{cases} \\
\theta(x, 0)=0 \forall x
\end{gathered}
$$

Sensitivity to $h_{\mathrm{L}}$ will be tested both in the theoretical case of zero friction and bottom slope, taking advantage of the analytical solution availability, and in a more realistic case with friction and bottom slope, using the numerical model.

2. The expression of $\mathbf{Q}$ is now derived for the case where the parameter $\psi$ is the friction coefficient $n$ : since $\mathbf{F}$ is not a function of $n, \partial \mathbf{F} / \partial \psi=0$ and

$$
\mathbf{Q}=\frac{\partial \mathbf{S}}{\partial \mathbf{U}} \mathbf{s}+\frac{\partial \mathbf{S}}{\partial n} \varepsilon_{n}
$$


with $(\partial \mathbf{S} / \partial \mathbf{U}) \mathbf{s}$ defined by Eq. (19) and

$$
\frac{\partial \mathbf{S}}{\partial n_{M}} \varepsilon_{n}=\left(\begin{array}{c}
0 \\
-2 g h \frac{S_{f}}{n_{M}} \varepsilon_{n}
\end{array}\right)
$$

where $\varepsilon_{n}=0$ in regions where the friction coefficient is unchanged and $\varepsilon_{n}=1$ in the regions where it contributes to the sensitivity (in the following, $\varepsilon_{n}$ will be taken equal to one over the whole domain). In this case, the initial condition on the water depth sensitivity $\eta$ will be nil if the initial water depth in the river $h_{\mathrm{R}}$ is prescribed to any value different from the normal height $h_{N}$, but non zero if $h_{\mathrm{R}}=h_{N}$, because $h_{N}$ (Eq. 17) depends on the friction coefficient:

$$
\begin{aligned}
& \text { if } h_{\mathrm{R}} \neq h_{N}: \eta(x, 0)=0 \forall x \\
& \text { if } h_{\mathrm{R}}=h_{N}: \eta(x, 0)= \begin{cases}0 & \text { for } x<x_{0} \\
\frac{3 \varepsilon_{n} h_{N}}{2 n_{M}} & \text { for } x>x_{0}\end{cases} \\
& \theta(x, 0)= \begin{cases}0 & \text { for } x<x_{0} \\
u_{\mathrm{R}} \eta(x, 0) & \text { for } x>x_{0}\end{cases}
\end{aligned}
$$

3. The sensitivity with respect to the bottom slope leads to:

$$
\mathbf{Q}=\frac{\partial \mathbf{S}}{\partial \mathbf{U}} \mathbf{s}+\frac{\partial \mathbf{S}}{\partial S_{0}} \varepsilon_{S_{0}}
$$

The derivative of $\mathbf{S}$ with respect to $\mathbf{U}$ remains the same as given in Eq.(19), and

$$
\frac{\partial \mathbf{S}}{\partial S_{0}} \varepsilon_{S_{0}}=\left(\begin{array}{c}
0 \\
g h \varepsilon_{S_{0}}
\end{array}\right)
$$

with $\varepsilon_{S_{0}}=1$ where $S_{0}$ contributes to the sensitivity. The initial conditions for the water depth sensitivity are non zero because, for a prescribed initial water elevation, a variation in the bottom slope will yield a variation in the water depth. Moreover, since the normal height depends on the bottom slope, an additional term appears when $h_{\mathrm{R}}=h_{N}$ :

$$
\begin{aligned}
\text { if } h_{\mathrm{R}} \neq h_{N}: \quad \eta(x, 0) & =\varepsilon_{S_{0}}\left(x-x_{0}\right) \forall x \\
\text { if } h_{\mathrm{R}}=h_{N}: \quad \eta(x, 0) & = \begin{cases}\varepsilon_{S_{0}}\left(x-x_{0}\right) \\
\varepsilon_{S_{0}}\left(x-x_{0}\right)-\frac{3 \varepsilon_{S_{0}} h_{N}}{4 S_{0}} & \text { for } x<x_{0} \\
\text { for } x>x_{0}\end{cases} \\
\theta(x, 0) & = \begin{cases}0 & \text { for } x<x_{0} \\
u_{\mathrm{R}} \eta(x, 0) & \text { for } x>x_{0}\end{cases}
\end{aligned}
$$

\section{Numerical application and results comparison}

\subsection{Flood propagation: sensitivity to the maximum flood discharge}

For this simplified representation of flood propagation in a river reach, parameters values given in Table 1 are used. After sufficient time for the steady state to be reached (Eq. 12), the input unit discharge rises by a factor 4 within ten minutes and then recedes to its initial value within 20 minutes.

Figure 3 shows the flow and sensitivity solution at 4 different simulation times. The standard deviations computed from both approaches for the water depth are plotted in figure 4 for a maximum input discharge $q_{\max }$ that follows a Beta law (Eq. 1) between 3.6 
and $4.4 \mathrm{~m}^{2} \mathrm{~s}^{-1}$ in figure $4 \mathrm{a}$ (i.e. for a possible relative variation of $\pm 10 \%$ ) and between 2.8 and $5.2 \mathrm{~m}^{2} \mathrm{~s}^{-1}$ in figure $4 \mathrm{~b}$ (i.e. for a possible relative variation of $\pm 30 \%$ ). It can be seen that standard deviation obtained from LSA and GUA are quite similar. To confirm this result, a convergence analysis is performed on the first two statistical moments (mean and standard deviation).

The relative error of $\mu_{L}$ to $\mu_{G}$ (resp. $\sigma_{L}$ to $\sigma_{G}$ ) computed from Eq. 4 (resp. 5 ) is shown in Figure 5 for the outputs $h$ and $q$ as a function of the possible percentage of variation of the maximum input discharge (from 10\% to 80\%). Because the local approach assumes linearity around a nominal value of the parameter of interest, $\mu_{L}, \sigma_{L}$ gets closer to $\mu_{G}, \sigma_{G}$ as the standard deviation of the input parameter distribution decreases, i.e. as the min-max interval is narrowed. Figure 5 a shows that the relative difference between the two means estimates $\left(\mu_{L}, \mu_{G}\right)$ remains smaller than $1.8 \%$ of GUA estimates for both water depth and unit discharge, even if the maximum input discharge parameter is allowed to vary by as much as $\pm 80 \%$, i.e. from $0.8 \mathrm{~m}^{2} \mathrm{~s}^{-1}$ to $7.2 \mathrm{~m}^{2} \mathrm{~s}^{-1}$. It can be seen in Figure $5 \mathrm{~b}$ that the estimation error of the standard deviation $\sigma_{L}$ obtained with LSA is always less than $5 \%$ of GUA results. This convergence analysis demonstrates that, even if the maximum input discharge is highly uncertain, the LSA approach enables an excellent reproduction of the output mean and standard deviation as obtained from 1000 Monte Carlo simulations. This was achieved in $80 \mathrm{~s}$ by LSA, compared to more than 12 hours for GUA in this test case.

\subsection{Dam-break problem: One-factor-at-a-time analysis}

\subsubsection{Theoretical case of no bottom slope and friction - sensitivity to initial reservoir water depth}

With the analytical solution for zero bottom slope and friction (Appendix B), the sensitivity analysis is performed with respect to the initial water depth in the reservoir $h_{\mathrm{L}}$. The flow and sensitivity solutions are computed for the parameters given in Table 2 with the water initially at rest (zero velocity) on both sides of the dam. A fixed value of $h_{\mathrm{R}}$ is prescribed for initial river water depth.

The initial water depth distribution is generated from $N=1000$ repetitions of the Beta law (Eq. 1), centered on the nominal value $h_{\mathrm{L}}^{0}=20$ and rescaled from $[0 ; 1]$ to the interval $[15 ; 25]$ (i.e. $\Delta \psi / \psi^{0}=25 \%$ ). Figure 6 shows the input distribution of the initial water depth $h_{\mathrm{L}}$ and the deciles of the output water depth and unit discharge obtained from the $N$ computations of the analytical solution at time $T_{\max }=5 \mathrm{~s}$.

Figure 7 shows the comparison between the two estimates of the output standard deviation $\sigma_{G}$ for the Monte Carlo simulations and $\sigma_{L}$ for the local method, using the analytical sensitivity solution for $\eta$ Eq. (B.10) and $\theta$ Eq (B.11) and Eq. (2). Results obtained with the local method shows steeper fronts than the Monte Carlo standard deviation and do not reproduce the strong dispersion near the shock. Indeed, the analytical solution of the sensitivity equations does not account for the sensitivity of the shock location. Consequently, the shock of the analytical solution is a Dirac, i.e. with a nil width and infinite amplitude. On the contrary, it can be seen in Figures $6 \mathrm{~b}$ and $6 \mathrm{c}$ (decile profiles) that the discontinuity location depends on the initial water depth in the reservoir, leading to a smoothed spike. Except for the difference in behavior across the shock, the standard deviation obtained by LSA is quite similar to GUA result everywhere else, while obtained after only one simulation instead of $N=1000$.

\subsubsection{Real-world test case}

A more realistic test case is built, that consists in a dam-break problem with bottom slope, friction and initial flow velocity in the river (see subsection 4.2). The initial water depth in the river $h_{\mathrm{R}}$ is taken as the normal depth $h_{N}$. The parameters used in this test case are given in Table 3. The uncertainty analysis is performed successively with respect to the main three parameters: the initial water depth in the reservoir $h_{\mathrm{L}}$, the bottom slope $S_{0}$ and 
the Manning's friction coefficient $n_{M}$. The local sensitivity is computed by solving Eqs (9) around nominal values of the parameters, which are given in Table 3 with superscript 0 and which are taken as reference values in the following. The samples used in the global method are generated by $N$ repetitions of the Beta law defined by Eq. (1), which are centered and rescaled with the parameters given in Table 4 . Computation time for $N=1000$ simulations is about $500 \mathrm{~s}$ compared to less than $1 \mathrm{~s}$ for the local method.

Output deciles obtained from the Monte Carlo simulations with respect to the initial reservoir water depth $h_{\mathrm{L}}$ are given in Figure 8. The local sensitivity solution $(\eta, \theta)$ is shown in Figure 9. Figure 10 compares the resulting standard deviation estimates from the GUA and LSA methods for uncertainty in the initial water depth in the dam (Figure 10a), the bottom slope (Figure 10b) and for the Manning's friction coefficient (Figure 10c). In all cases, we can see proper reproduction of the global standard deviation output using the local sensitivity in conjunction with Eq.(2), over the whole domain but the shock area where the spike is always sharper with the local approach.

These results were obtained for a given magnitude of relative variation in each input parameter: from $\Delta \psi=0.2 \psi^{0}$ for water depth to $\Delta \psi=0.5 \psi^{0}$ for bottom slope (see Table 4 ). A convergence analysis was performed to investigate how the difference between the two methods behaves as a function of $\Delta \psi / \psi^{0}$. This analysis is shown for the output water depth mean and standard deviation in the case of an uncertain initial water depth in the reservoir (Figure 11), but conclusions remain valid for the the unit discharge $q$ and/or with respect to other parameters. The relative error in mean and standard deviation of output $h(x)$ estimated from LSA with respect to GUA is calculated by equations (5) and (4).

As expected $\left(\mu_{L}, \sigma_{L}\right)$ converges to $\left(\mu_{G}, \sigma_{G}\right)$ as the standard deviation of the input parameter distribution gets smaller, i.e. as the min-max interval is narrower. The relative discrepancy between output means from the two methods remains smaller than $5 \%$ (Figure 11a) even for $\Delta \psi / \psi^{0}=80 \%$, which corresponds to a standard deviation of the initial water elevation in the reservoir of $\sigma_{z_{\mathrm{L}}}=2.42 \mathrm{~m}$. Because of the strong over-estimation of the spike amplitude across the discontinuity, the relative error in output standard deviation can reach more than $30 \%$ when $\Delta \psi / \psi^{0}=80 \%$ (Figure 11b). However, when excluding a $4 \mathrm{~m}$ interval centered on the shock from the domain over which the error is computed, the relative difference between LSA and GUA remains smaller than 9\%. This illustrates that uncertainty is properly estimated by the local method over the whole domain but the immediate vicinity of the shock.

\subsection{Dam-break problem: three-factors-at-a-time}

The simultaneous, independant variation of the main three parameters: $z_{\mathrm{L}}, S_{0}$ and $n_{M}$ was considered. The number of simulations was set to $N=10000$, corresponding to a computational time of about one hour. For each simulation, each of the three parameters was generated independently using a beta law centered on the nominal values $\psi^{0}$ and bounded by a min-max interval $\left[\psi^{0}-\Delta \psi ; \psi^{0}+\Delta \psi\right]$ where $\Delta \psi$ is chosen as a given percentage of $\psi^{0}$, identical for the three parameters.

Figure 12 compares the standard deviation estimates by the global and local approaches for water depth along the river reach, when the parameters are allowed to vary from their nominal value by a percentage in the range $\pm 0.1 \%, \pm 20 \%$ or $\pm 40 \%$, successively. In the first case (Figure 12a), the reproduction by the LSA approach is nearly identical to the GUA standard deviation. However, when the standard deviation in the input parameter increases (Figure 12b and 12c), while the two approaches remain very similar over most of the domain, they behave differently around the flow discontinuity: the spike amplitude from the local approach increases with the input variance but remains narrow; on the contrary, the standard deviation of the global approach decreases in amplitude across the discontinuity but becomes wider. Despite this difference, the spike integral remains rather similar between the two methods. Indeed, the relative difference between the standard deviation integral from the 
local approach $A_{L}$ and the one from the global approach $A_{G}$, calculated as $\left|A_{L}-A_{G}\right| / A_{G}$, remains smaller than 0.13 even for the maximum variation of the three parameters $( \pm 40 \%)$.

As in previous cases, a convergence analysis was performed, for the water depth. Figure 11 shows the relative errors in the first statistical moments computed from LSA with respect to GUA (Eqs.5 and4) as a function of $\Delta \psi / \psi^{0}$. In Figure 11c and d, the relative error, computed over the whole domain reaches $3 \%$ for the mean and $18 \%$ for the standard deviation when the three parameters are allowed to vary from their nominal value by $\pm 0.1 \%$ to $\pm 40 \%$. The relative error obtained after excluding the same $4 \mathrm{~m}$ across the shock as previously remains smaller than $1.6 \%$ and $4.5 \%$ of GUA estimates even when the min-max interval of the input distribution reaches as much as $\pm 0.4 \psi^{0}$ for each parameter simultaneously.

An interesting feature of the local sensitivity approach, in the ubiquitous case of multiple uncertain parameters, is that it directly provides for first-order estimation of each parameter's importance in the generation of the overall model output uncertainty. Indeed, Eqs (3), expressing the output variance as a sum of contributions from each uncertain parameter, immediately translates into relative shares of each of them in the resulting total uncertainty, through division of each contribution by their sum.

Applying this rule to the above numerical case, shows that the area of predominance is different for each parameter (Figure 13): water elevation $h_{\mathrm{L}}$ is the most important parameter upstream of the shock (but, not unexpectedly, has no effect downstream), while the friction coefficient $n$ is the most important one downstream of the shock.

Finally, should the uncertain parameters not be considered independent, a similar analysis, including additionnal, covariance terms in Eqs (3), could be further developed.

\section{Conclusion}

The main advantage of the proposed equation-based, local approach (LSA) is that it requires only a single simulation instead of many hundreds or thousands for the global method (GUA), leading to a considerable gain in computational time. This study shows that the local sensitivity can be successfully used in place of a large number of Monte Carlo simulations for some complex, nonlinear hydrodynamic problems, wherever the flow remains continuous.

This is the case for instance of the propagation of flash floods, or of the dam-break problem outside a very small area around the shock that propagates downstream. For this latter problem, estimating the spike in variance around the flow discontinuity remains a problem. This was to be expected because the local and global estimations of the output standarddeviation can be identical only if (i) the output function is a continuous function of the parameters, and (ii) the function can be assumed quasi-linear within the range of variation of the parameters. The accuracy of the local method anywhere the flow is continuous suggests the interesting finding that hydrodynamics of river flow is generally only very mildly non-linear, even for such abrupt processes as flash floods or dam failure. However, where and when the flow becomes discontinuous, both assumptions above are obviously violated.

A treatment of the moving shock has been proposed $[8,13]$ to eliminate the spike in the local sensitivity solution. However, this approach is not appropriate for the applications considered in this paper because it misses the mode in variance observed in the GUA output standard deviation across the discontinuity. Further research should thus be devoted to designing methods that allow for better approximation by the local approach of the output standard deviation near flow discontinuities. Despite this difficulty, the relative error produced by LSA compared to Monte Carlo simulations including the shock area remains smaller than $18 \%$ when all three parameters vary concurrently by as much as $\pm 40 \%$. Local sensitivity analysis thus appears as a suitable approach to uncertainty analysis in the range of parameter variation classically experienced for the shallow water equations. Moreover, 
that the standard deviation integral is largely preserved between the local and the global approach suggests that reliable estimation of the spike could possibly be obtained. Finally, beyond the computing performance advantage of the local approach, an additional benefit lies in the valuable information that it readily brings with respect to the relative importance of multiple uncertain parameters in total model uncertainty. 


\begin{tabular}{lll}
\hline Symbol & Definition & Value \\
\hline$g$ & Gravitational acceleration & $9.81 \mathrm{~m} \mathrm{~s}^{-2}$ \\
$L$ & Length of the simulation domain & $3000 \mathrm{~m}$ \\
$S_{0}$ & Bottom slope & $10^{-3}$ \\
$n_{M}$ & Manning's friction coefficient & $0.025 \mathrm{~m}^{-1 / 3} \mathrm{~s}$ \\
$q_{\mathrm{up}}$ & Unit discharge prescribed at the upstream boundary & see Fig. 1 and Eq. $(12)$ \\
$q_{\max }$ & Maximum discharge prescribed at the upstream boundary & $4 \mathrm{~m}^{2} \mathrm{~s}^{-1}$ \\
$q_{\min }$ & Minimum discharge prescribed at the upstream boundary & $1 \mathrm{~m}^{2} \mathrm{~s}^{-1}$ \\
$t_{1}$ & Time of flood beginning & $0 \mathrm{~min}$ \\
$t_{2}$ & Time of maximum input discharge $\left(q_{\mathrm{b}}=q_{\max }\right)$ & $10 \mathrm{~min}$ \\
$t_{3}$ & End of flood $\left(q_{\mathrm{b}}=q_{\min }\right)$ & $30 \mathrm{~min}$ \\
$\theta_{\mathrm{up}}$ & Unit discharge sensitivity prescribed at the upstream boundary & see Fig. 1 \\
$\mathrm{~F}_{\mathrm{ds}}$ & Froude number prescribed at the downstream boundary & 0.8 \\
$T_{\max }$ & Final simulation time for methods comparison & $15 \mathrm{~min}$ \\
$\Delta x$ & Cells width used for the discretization & $1 \mathrm{~m}$ \\
$N$ & Number of Monte Carlo simulations & 1000 \\
\hline
\end{tabular}

Table 1: Parameters for the flood propagation test case.

\begin{tabular}{lll}
\hline Symbol & Definition & Value \\
\hline$g$ & Gravitational acceleration & $9.81 \mathrm{~m} \mathrm{~s}^{-2}$ \\
$L$ & Length of the simulation domain & $200 \mathrm{~m}$ \\
$x_{0}$ & Initial location of the dam & $100 \mathrm{~m}$ \\
$h_{\mathrm{L}}$ & Initial water depth in the reservoir, on the left-hand side of the dam & $20 \mathrm{~m}$ \\
$\eta_{\mathrm{L}}$ & Initial sensitivity of the water depth in the reservoir & 1 \\
$h_{\mathrm{R}}$ & Initial water depth in the river, on the right-hand side of the dam & $2 \mathrm{~m}$ \\
$\eta_{\mathrm{R}}$ & Initial sensitivity of the water depth in the river & 0 \\
$q_{\mathrm{L}, \mathrm{R}}$ & Initial unit discharge on the left- and right-hand sides of the dam & $0 \mathrm{~m}^{2} \mathrm{~s}^{-1}$ \\
$\theta_{\mathrm{L}, \mathrm{R}}$ & Initial sensitivity of unit discharge on the left- and right-hand sides of the dam & $0 \mathrm{~m}^{2} \mathrm{~s}^{-1}$ \\
$S_{0}$ & Bottom slope & 0 \\
$n_{M}$ & Manning's friction coefficient & $0 \mathrm{~m}^{-1 / 3} \mathrm{~s}$ \\
$T_{\max }$ & Final simulation time & $5 \mathrm{~s}$ \\
$(\Delta x)_{r}$ & Cells width used for the discretization & $0.1 \mathrm{~m}$ \\
$N$ & Number of Monte Carlo simulations & 1000 \\
\hline
\end{tabular}

Table 2: Parameters for the dam-break problem without friction and slope.

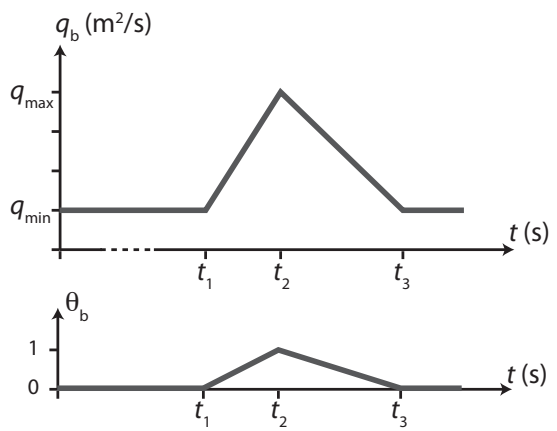

Figure 1: Flood propagation test case. Unit discharge and sensitivity prescribed at the upstream boundary. 


\begin{tabular}{lll}
\hline Symbol & Definition & Value \\
\hline$g$ & Gravitational acceleration & $9.81 \mathrm{~m} \mathrm{~s}^{-2}$ \\
$L$ & Length of the simulation domain & $200 \mathrm{~m}$ \\
$x_{0}$ & Initial position of the dam & $100 \mathrm{~m}$ \\
$T_{\max }$ & Final simulation time & $3 \mathrm{~s}$ \\
$h_{\mathrm{R}}$ & Initial water depth in the river (right side of the dam) & $h_{N}$ Eq. $(17)$ \\
$u_{\mathrm{R}}$ & Initial flow velocity in the river & $1 \mathrm{~m} \mathrm{~s}^{-1}$ \\
$\Delta x$ & Cells width used for the discretization & $0.1 \mathrm{~m}$ \\
$z_{\mathrm{L}}^{0}$ & Initial water elevation in the reservoir & $10 \mathrm{~m}$ \\
$S_{0}^{0}$ & Bottom slope & $1 \cdot 10^{-2}$ \\
$n_{M}^{0}$ & Manning's friction coefficient & $0.025 \mathrm{~m}^{-1 / 3} \mathrm{~s}$ \\
$\Delta \psi$ & Defines the possible parameter variation & $\psi \in\left[\psi^{0}-\Delta \psi, \psi^{0}+\Delta \psi\right]$ \\
$N$ & Number of Monte Carlo simulations & 1000 \\
\hline
\end{tabular}

Table 3: Problem parameters for the "real-world" dam-break problem. The superscript 0 denotes the nominal values for the local sensitivity approach, which are also used as the distribution means in the Monte Carlo simulations (see Table 4).

\begin{tabular}{l|lll|ll}
\hline Parameter & Mean & Minimum & Maximum & $\Delta \psi$ & Standard deviation $\sigma$ \\
\hline$z_{\mathrm{L}}(\mathrm{m})$ & $z_{\mathrm{L}}^{0}=10$ & 8 & 12 & $0.2 z_{\mathrm{L}}^{0}$ & 0.598 \\
$n_{M}\left(\mathrm{~m}^{-1 / 3} \mathrm{~s}\right)$ & $n_{M}^{0}=0.025$ & 0.015 & 0.035 & $0.4 n_{M}^{0}$ & $2.96 \cdot 10^{-3}$ \\
$S_{0}(-)$ & $S_{0}^{0}=1 \cdot 10^{-2}$ & $0.5 \cdot 10^{-2}$ & $1.5 \cdot 10^{-2}$ & $0.5 S_{0}^{0}$ & $1.49 \cdot 10^{-3}$ \\
\hline
\end{tabular}

Table 4: Dam-break problem, one-factor-at-a-time: Characteristics of the parameter distributions for the Monte Carlo analysis. Each distribution is centered on the nominal value (0 superscript) of Table 3 .
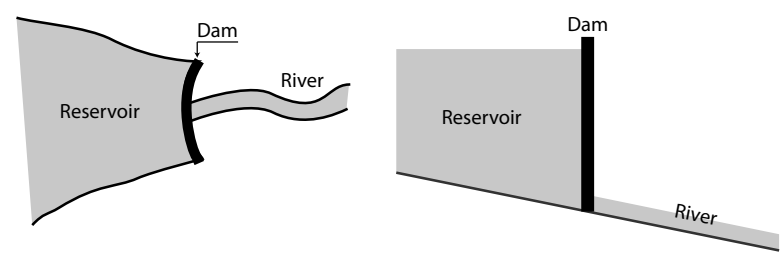

Figure 2: Schematic view of the dam. Left: view from above; right: longitudinal crosssection. 


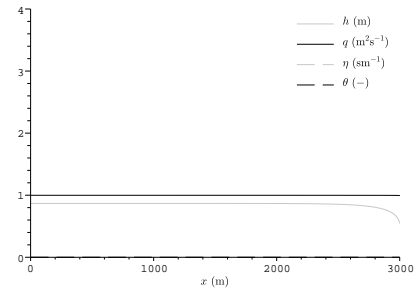

(a) $t=0 \mathrm{~min}$

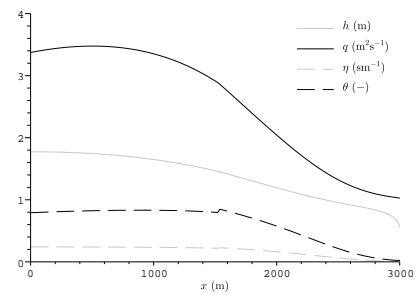

(c) $t=15 \mathrm{~min}$



(b) $t=10 \mathrm{~min}$

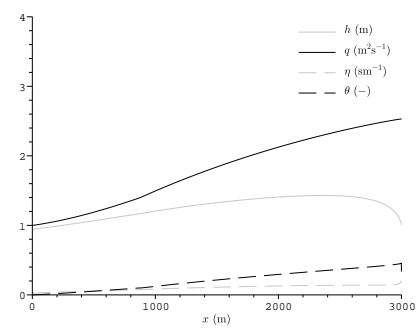

(d) $t=30 \min$

Figure 3: Flood propagation: water depth, unit discharge and their sensitivity with respect to the maximum input discharge at four times.
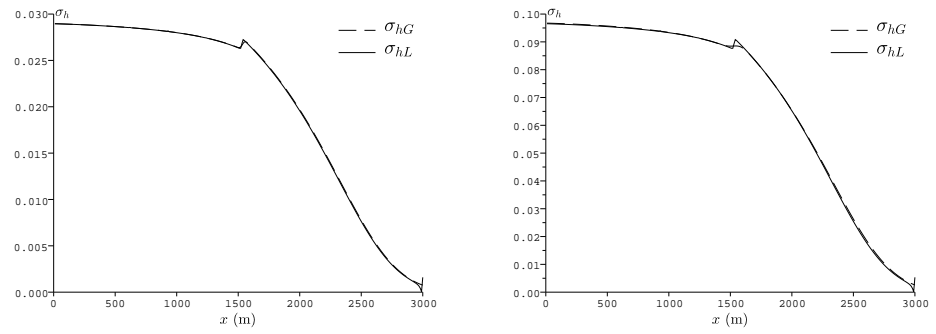

Figure 4: Flood propagation test case. Estimation of the standard deviation $\sigma_{h}(x)$ of the output water depth at time $T_{\max }$ using the local approach $\sigma_{h L}$ and the global approach $\sigma_{h G}$; with a maximum relative variation of maximum input discharge $\Delta q_{\max } / q_{\max }=10 \%$ (left) and 30\% (right). 

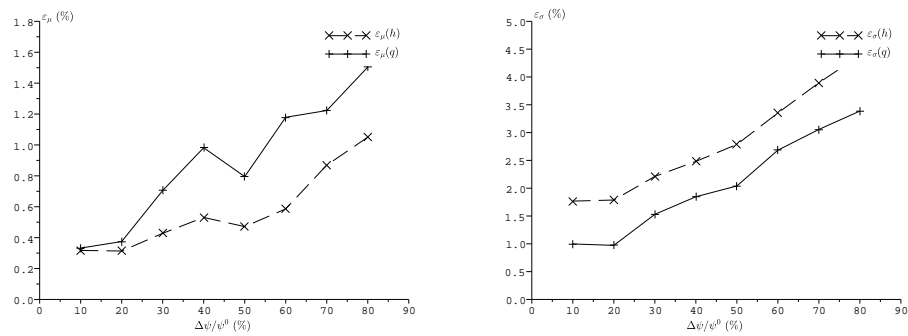

Figure 5: Convergence analysis for the flood propagation test case. Relative departures from the local to the global approach (Eqs $(4)(5))$ for the water depth $h$ and discharge $q$ means (left) and standard deviations (right), depending on relative parameter variability $\Delta \psi / \psi^{0}$. Differences remain smaller than $1.8 \%$ (resp. $5 \%$ ) even when the maximum input discharge is allowed to vary by $80 \%$ (i.e. from $0.8 \mathrm{~m}^{2} \mathrm{~s}^{-1}$ to $7.2 \mathrm{~m}^{2} \mathrm{~s}^{-1}$ ).

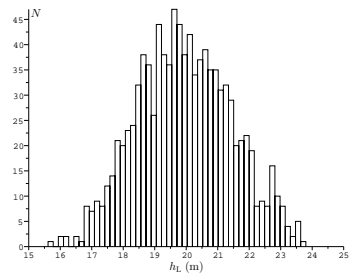

(a)

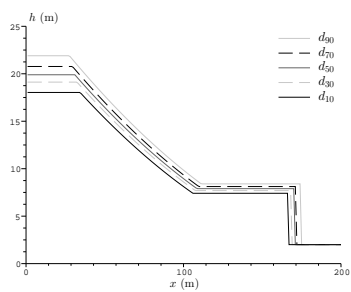

(b)

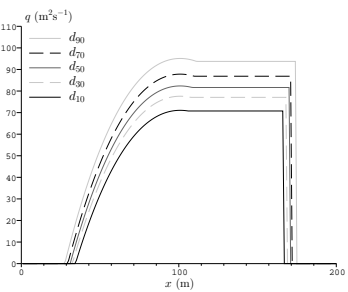

(c)

Figure 6: Monte Carlo simulations: a) distribution of parameter $h_{\mathrm{L}}$ obtained from random sampling within a Beta distribution (Eq. 1); b) and c) deciles of the output water depth and unit discharge at time $T_{\max }$.
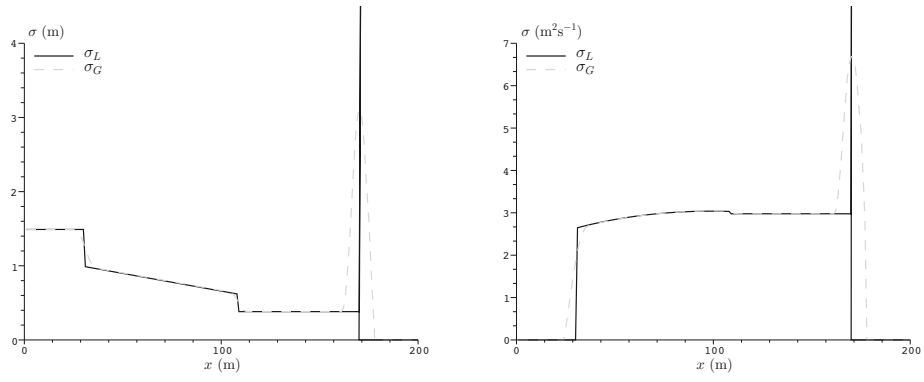

Figure 7: Analytical solution at time $T_{\max }$. Estimation of the standard deviation $\sigma(x)$ of the output: water depth (left) and unit discharge (right). $\sigma_{G}$ is estimated from 1000 simulations of the analytical flow solution (B.4) and $\sigma_{L}$ from the analytical sensitivity solutions (B.10)-(B.11) using Eq. (2). The analytical solution for the sensitivity does not account for the sensitivity of the shock location. 

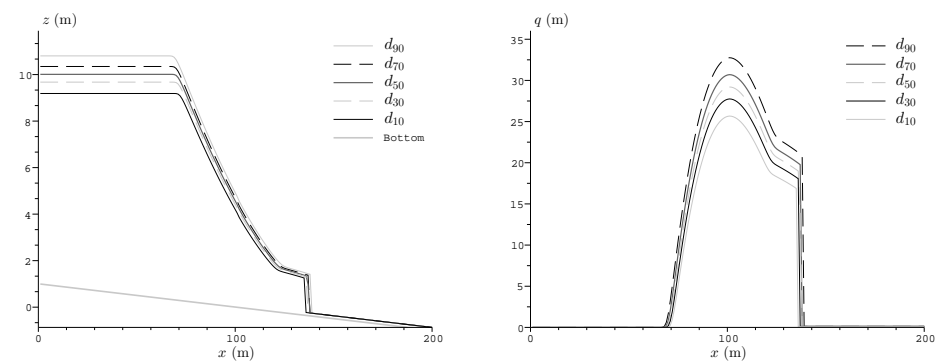

Figure 8: Dam-break problem, one-factor-at-a-time: initial water depth in the reservoir $z_{L}$. Deciles of the output distributions of $h$ and $q$ obtained from the Monte Carlo simulations with an input distribution of the initial water elevation in the reservoir, $z_{\mathrm{L}}$.
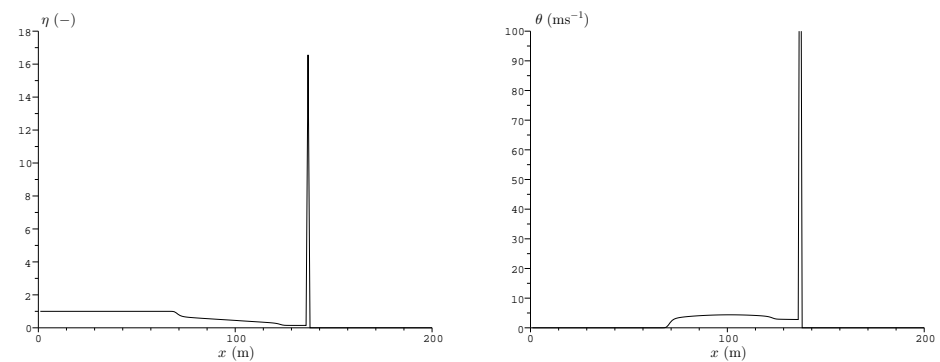

Figure 9: One-factor-at-a-time: initial water depth in the reservoir $z_{L}$. Local sensitivity of $h$ and $q$ with respect to the initial water elevation in the reservoir, $z_{\mathrm{L}}$. 



(a)


(b)
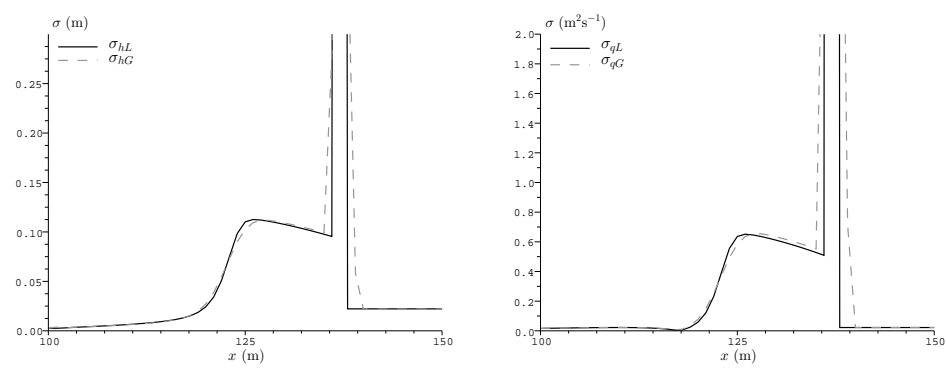

(c)

Figure 10: Dam-break problem, one-factor-at-a-time. Comparison between the standard deviations of the outputs $h$ (left) and $q$ (right), estimated from the global $\sigma_{G}$ and local $\sigma_{L}$ approaches for parameters: a) initial water depth in the reservoir $z_{L}$, b) bottom slope $S_{0}$ and c) friction coefficient $n_{M}$. 


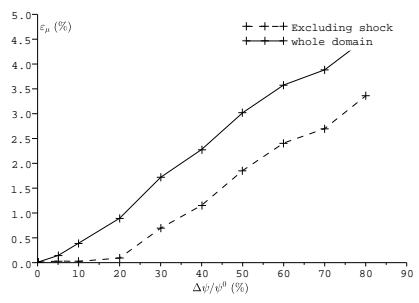

(a) OAT: Output mean

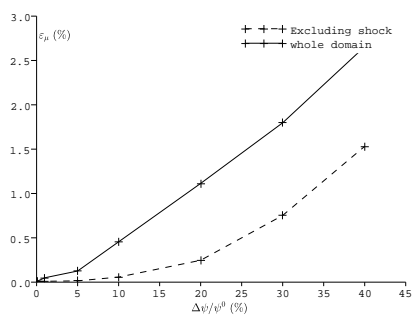

(c) 3AT: Output mean

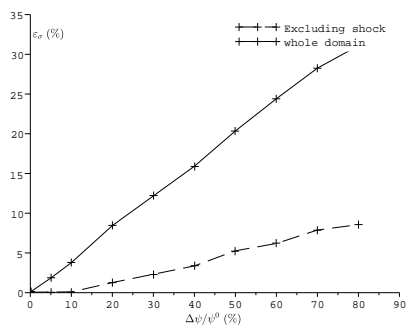

(b) OAT: Output standard deviation

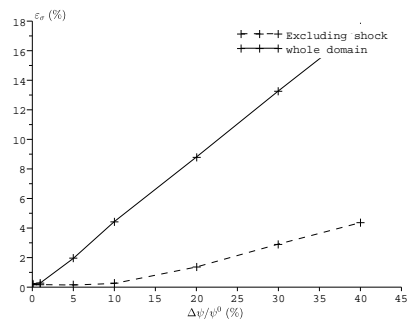

(d) 3AT: Standard deviation

Figure 11: Dam-break problem, one (up) or three (down) factor(s)-at-a-time: convergence analysis for initial water elevation in reservoir $z_{\mathrm{L}}$. Relative errors (Eqs (4)(5)) on estimates of first statistical moments of water depth $h$ by LSA compared to GUA, as a function of relative parameter variability $\Delta \psi / \psi^{0}$ : a, c) for the output mean; b, d) for the output standard deviation including the whole domain or excluding $4 \mathrm{~m}$ across the shock.

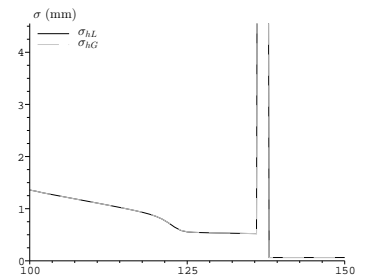

(a) $\psi \in \psi^{0} \pm 0.1 \%$

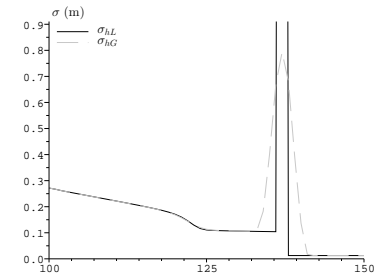

(b) $\psi \in \psi^{0} \pm 20 \%$

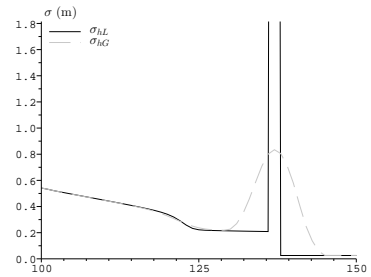

(c) $\psi \in \psi^{0} \pm 40 \%$

Figure 12: Dam-break problem, three-factors-at-a-time. Estimated standard deviations in output $h$ as a function of $x$ (zoom near the shock location), from global and local methods, for three different magnitudes of variation in the three parameters $\psi=z_{L}, S_{0, n_{M}}$. Parameters $\psi$ are taken from a distribution of 10000 repetitions of three Beta laws centered on $\psi_{0}$ and included in $\psi_{0} \pm X \%$ with $X=0.1,20$ and 40 . When the input variance increases, the output variance remains correctly reproduced by the local approach in the whole domain except around the discontinuity where the spike amplitude is highly overestimated. 


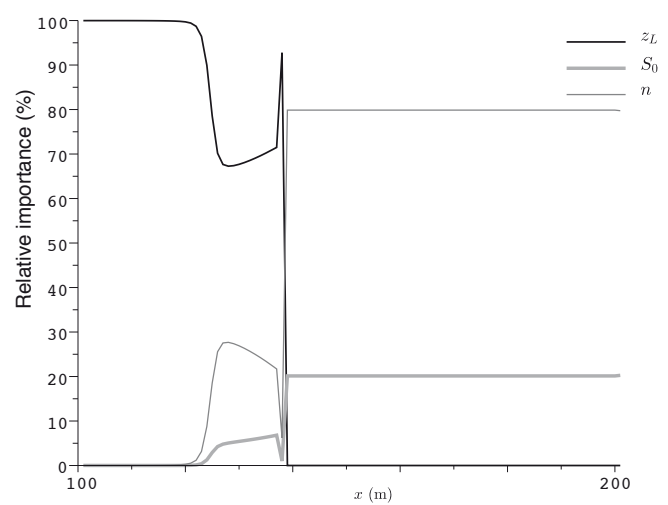

Figure 13: Dam-break problem, three-factors-at-a-time. Relative importance of the three parameters along the river reach at time $T_{\max }$ after dam break (estimated by LSA method). 


\section{Appendix A. Notations}

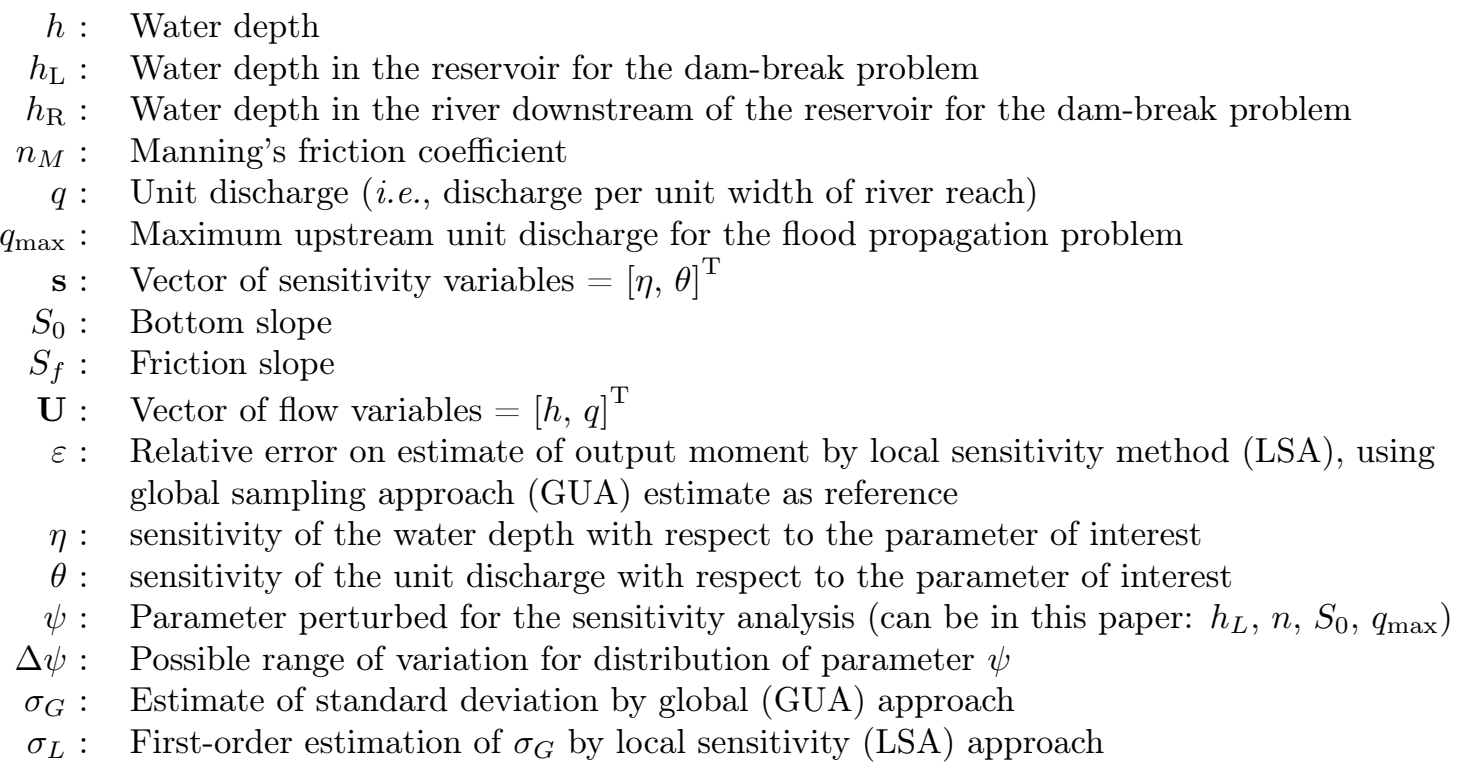

\section{Appendix B. Analytical solution of the dam-break problem without source term}

The analytical solution for the flow (6) and sensitivity (9) equations can be obtained for the dam-break problem when no friction and bottom slope are considered, by solving the following Riemann problem:

$$
\begin{aligned}
\frac{\partial \mathbf{U}(x, t)}{\partial t}+\frac{\partial \mathbf{F}(x, t)}{\partial x} & =0 \\
\frac{\partial \mathbf{s}(x, t)}{\partial t}+\frac{\partial \mathbf{G}(x, t)}{\partial x} & =0 \\
(\mathbf{U}, \mathbf{s})(x, 0) & = \begin{cases}\left(\mathbf{U}_{\mathbf{L}}, \mathbf{s}_{\mathbf{L}}\right) & \text { for } x<x_{0} \\
\left(\mathbf{U}_{\mathbf{R}}, \mathbf{s}_{\mathbf{R}}\right) & \text { for } x>x_{0}\end{cases}
\end{aligned}
$$

where $x_{0}$ is the abscissa of the dam.

The general solution of this Riemann problem is known to be made of two waves with opposite directions separated by an intermediate region of constant state. The first one is a rarefaction wave while the second one is a shock wave (see Figure B.14). The waves celerities are given by

$$
\left(\begin{array}{c}
\lambda^{-} \\
\lambda^{+}
\end{array}\right)=\left(\begin{array}{c}
u-c \\
u+c
\end{array}\right)
$$

The comprehensive development of the analytical solution can be found for example in $[8,13]$. The solution is self-similar [19], that is, it depends only on the variable $\xi=\left(x-x_{0}\right) / t$. The flow solution is given by:

$$
h=\left\{\begin{array}{lll}
h_{\mathrm{L}} & \text { if } & \xi<(u-c)_{\mathrm{L}} \\
h_{r}(\xi) & \text { if } \quad(u-c)_{\mathrm{L}}<\xi<(u-c)_{*} \\
h_{*} & \text { if } \quad(u-c)_{*}<\xi<c_{s} \\
h_{\mathrm{R}} & \text { if } \quad c_{s}<\xi
\end{array}\right.
$$




$$
q=\left\{\begin{array}{lll}
q_{\mathrm{L}} & \text { if } & \xi<(u-c)_{\mathrm{L}} \\
h_{r} u_{r}(\xi) & \text { if } & (u-c)_{\mathrm{L}}<\xi<(u-c)_{*} \\
h_{*} u_{*} & \text { if } & (u-c)_{*}<\xi<c_{s} \\
q_{\mathrm{R}} & \text { if } & c_{s}<\xi
\end{array}\right.
$$

where $c_{r}, h_{r}, u_{r}$ are the expressions of $c, h$ and $u$ across the rarefaction wave:

$$
\begin{gathered}
c_{r}=\frac{1}{3}\left(u_{\mathrm{L}}+2 c_{\mathrm{L}}-\xi\right) \\
h_{r}=c_{r}^{2} / g \\
u_{r}=\frac{1}{3}\left(u_{\mathrm{L}}+2 c_{\mathrm{L}}+2 \xi\right)
\end{gathered}
$$

$c_{*}$ and $u_{*}$ are the expressions of $c$ and $u$ in the internal region of constant state:

$$
\begin{aligned}
c_{*} & =\sqrt{g h_{*}} \\
u_{*} & =\left(u_{\mathrm{L}}+2 c_{\mathrm{L}}-2 c_{*}\right)
\end{aligned}
$$

The shock speed $c_{s}$ and the water depth in the internal region of constant state $h_{*}$ are obtained by solving the jump relationships $(2 \times 2$ system of non-linear equations) across the shock:

$$
\mathbf{F}_{*}-\mathbf{F}_{\mathbf{R}}=\left(\mathbf{U}_{*}-\mathbf{U}_{\mathbf{R}}\right) c_{s}
$$

The first equation of (B.8) yields

$$
c_{s}=\frac{h_{*} u_{*}-q_{\mathrm{R}}}{h_{*}-h_{\mathrm{R}}}
$$

and $h_{*}$ is obtained by solving iteratively the second equation of (B.8).

The sensitivity solution is obtained by differentiating (B.3) and (B.4) with respect to $\psi$ :

$$
\begin{gathered}
\eta=\left\{\begin{array}{lll}
\eta_{\mathrm{L}} & \text { if } & \xi<(u-c)_{\mathrm{L}} \\
\eta_{r}=\frac{2}{g} c_{r} \chi_{r} & \text { if } & (u-c)_{\mathrm{L}}<\xi<(u-c)_{*} \\
\eta_{*} & \text { if } & (u-c)_{*}<\xi<c_{s} \\
\eta_{\mathrm{R}} & \text { if } & c_{s}<\xi
\end{array}\right. \\
\theta=\left\{\begin{array}{lll}
\theta_{\mathrm{L}} & \text { if } & \xi<(u-c)_{\mathrm{L}} \\
(\eta u+h \nu)_{r} & \text { if } & (u-c)_{\mathrm{L}}<\xi<(u-c)_{*} \\
\theta_{*} & \text { if } & (u-c)_{*}<\xi<c_{s} \\
\theta_{\mathrm{R}} & \text { if } & c_{s}<\xi
\end{array}\right.
\end{gathered}
$$

where $\chi$ and $\nu$ are the sensitivities of $c$ and $u$ with respect to the parameter $\psi$. Across the rarefaction wave, these sensitivities are identical and given by:

$$
\chi_{r}=\nu_{r}=\left[\frac{1}{3 h}(\theta-u \eta+c \eta)\right]_{\mathrm{L}}
$$

The sensitivities $\eta_{*}$ and $\theta_{*}$ in the internal region of constant state are found by solving the generalisation of the jump relationships to the sensitivity:

$$
\mathbf{G}_{*}-\mathbf{G}_{\mathbf{R}}=\left(\mathbf{s}_{*}-\mathbf{s}_{\mathbf{R}}\right) c_{s}+\left(\mathbf{U}_{*}-\mathbf{U}_{\mathrm{R}}\right) \frac{\partial c_{s}}{\partial h_{\mathrm{L}}}
$$



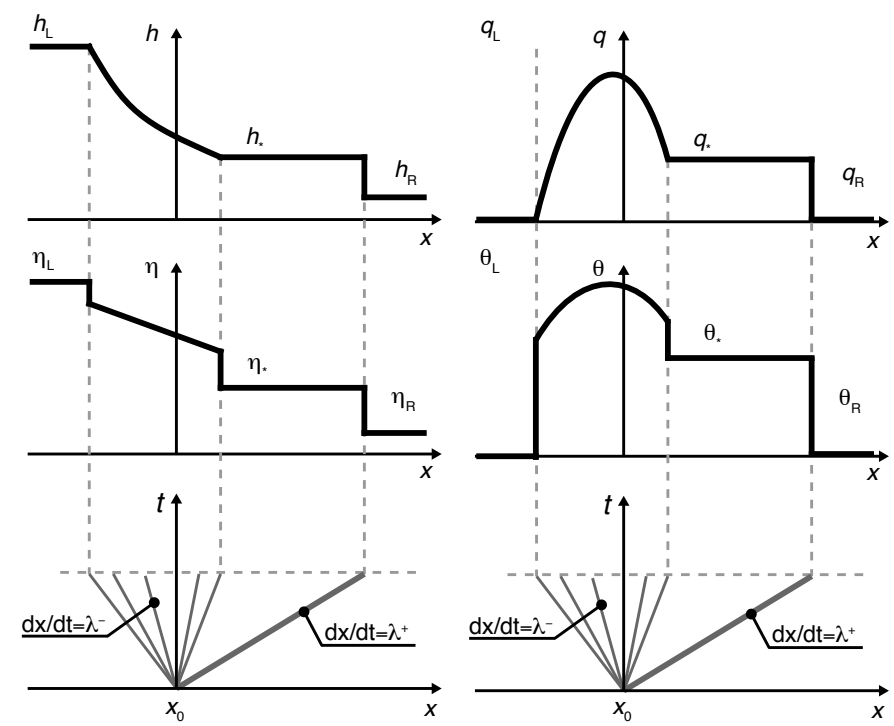

Figure B.14: Structure of the flow and sensitivity solutions for the dam-break problem. Left: water depth $h$ and its sensitivity $\eta$. Right: unit discharge $q$ and its sensitivity $\theta$.

\section{References}

[1] C. Bardos and O. Pironneau. A formalism for the differentiation of conservation laws. Comptes Rendus de l'Académie des Sciences Paris, Ser. I, 335:839-845, 2002.

[2] K. Beven. Prophecy, reality and uncertainty in distributed hydrological modeling. Advances in Water Resources, 16(1):41-51, 1993.

[3] K. Beven and J. Freer. Equifinality, data assimilation, and uncertainty estimation in mechanistic modelling of complex environmental systems using the GLUE methodology. Journal of Hydrology, 249(1-4):11-29, Aug. 2001.

[4] E. Borgonovo. Measuring uncertainty importance: Investigation and comparison of alternative approaches. Risk Analysis, 26(5):1349-1361, 2006.

[5] E. Borgonovo. A new uncertainty importance measure. Reliability Engineering $\mathscr{6}$ System Safety, 92(6):771 - 784, 2007.

[6] V. T. Chow. Open-channel hydraulics. Blackburn Press, 1959.

[7] C. Delenne, P. Finaud-Guyot, V. Guinot, and B. Cappelaere. Sensitivity of the 1D shallow water equations with source terms: Solution method for discontinuous flows. International Journal for Numerical Methods in Fluids, 67(8):981-1003, In press.

[8] C. Delenne, V. Guinot, and B. Cappelaere. Direct sensitivity computation for the Saint-Venant equations with hydraulic jumps. Comptes Rendus Mécanique, 336:766$771,2008$.

[9] H. Fang, M. Rais-Rohani, Z. Liu, and M.F. Horstemeyer. A comparative study of metamodeling methods for multiobjective crashworthiness optimization. Computers $\&$ Structures, 83(25-26):2121 - 2136, 2005.

[10] G. Freni, G. Mannina, and G. Viviani. Uncertainty in urban stormwater quality modelling: The influence of likelihood measure formulation in the GLUE methodology. Science of the Total Environment, 408(1):138-145, Dec. 2009. 
[11] V. Guinot and B. Cappelaere. Sensitivity equations for the one-dimensional shallow water equations: Practical application to model calibration. Journal of Hydrologic Engineering, 14:858-861, 2009.

[12] V. Guinot, B. Cappelaere, and C. Delenne. Finite volume solution of the onedimensional shallow water sensitivity equations. Journal of Hydraulic Research, 47(6):811-819, 2009.

[13] V. Guinot, C. Delenne, and B. Cappelaere. An approximate-state Riemann solver for sensitivity equations with discontinuous solutions. Advances in Water Resources, 32(1):61-77, 2009.

[14] M. D. Gunzburger. Sensitivities, adjoints and flow optimization problems. International Journal for Numerical Methods in Fluids, 31:53-78, 1999.

[15] J.W. Hall, S. Tarantola, P.D. Bates, and M.S. Horritt. Distributed sensitivity analysis of flood inundation model calibration. Journal of Hydraulic Engineering-ASCE, 131(2):117-126, 2005.

[16] J.C. Helton, J.D. Johnson, C.J. Sallaberry, and C.B. Storlie. Survey of sampling-based methods for uncertainty and sensitivity analysis. Reliability Engineering 83 System Safety, 91(10-11):1175 - 1209, 2006. The Fourth International Conference on Sensitivity Analysis of Model Output (SAMO 2004) - SAMO 2004.

[17] R. Jin, X. Du, and W. Chen. The use of metamodeling techniques for optimization under uncertainty. Structural and Multidisciplinary Optimization, 25:99-116, 2003. $10.1007 / \mathrm{s} 00158-002-0277-0$.

[18] A. L. Kay and H. N. Davies. Calculating potential evaporation from climate model data: A source of uncertainty for hydrological climate change impacts. Journal of Hydrology, 358(3-4):221-239, Sep. 2008.

[19] P.-D. Lax. Hyperbolic systems of conservation laws. Communications on Pure and Applied Mathematics, 10:537-566, 1957.

[20] N. Mazzilli, V. Guinot, and H. Jourde. Sensitivity analysis of two-dimensional steadystate aquifer flow equations. implications for groundwater flow model calibration and validation. Advances in Water Resources, 33:905-922, 2010.

[21] M. D. McKay, R.J. Beckman, and W.J Conover. A comparison of three methods of selecting values of input variables in the analysis of output from a computer code. Technometrics, 21:239-245, 1979.

[22] M. Minville, F. Brissette, and R. Leconte. Uncertainty of the impact of climate change on the hydrology of a nordic watershed. Journal of Hydrology, 358(1-2):70-83, Aug. 2008.

[23] F. Pappenberger, P. Matgen, K. J. Beven, J.-B. Henry, L. Pfister, and P. De Fraipont. Influence of uncertain boundary conditions and model structure on flood inundation predictions. Advances in Water Resources, 29(10):1430-1449, Oct. 2006.

[24] H. Roux and D. Dartus. Sensitivity analysis and predictive uncertainty using inundation observations for parameter estimation in open-channel inverse problem. Journal of Hydraulic Engineering-ASCE, 134(5):541-549, 2008.

[25] A. Saltelli, P. Annoni, I. Azzini, F. Campolongo, M. Ratto, and S. Tarantola. Variance based sensitivity analysis of model output. design and estimator for the total sensitivity index. Computer Physics Communications, 181:259-270, 2010. 
[26] A. Saltelli, K. Chan, and E.M Scott. Sensitivity analysis. Probability and Statistics series. John Wiley \& Sons publishers, 2000.

[27] A. Saltelli and S. Tarantola. On the relative importance of input factors in mathematical models: safety assessment for nuclear waste disposal. Journal of American Statistical Association, 97:702-709, 2002.

[28] A. Serrat-Capdevila, J. B. Valdes, J. Perez Gonzalez, K. Baird, L. J. Mata, and T. Maddock III. Modeling climate change impacts and uncertainty on the hydrology of a riparian system: The San Pedro Basin (Arizona/Sonora). Journal of Hydrology, 347(12):48-66, DEC 152007.

[29] J. J. Stoker. Water Waves. Interscience, Wiley, New-York, 1957. 